\title{
Explorando uma colmeia: uma proposta de modelagem com o GeoGebra 3D
}

\author{
Débora Silva Soares, PPGEMAT/IME/UFRGS, debora.soares@ufrgs.br \\ Márcia Rodrigues Notare, PPGEMAT/IME/UFRGS, marcia.notare@ufrgs.br \\ Matheus Faria de Moraes, IME/UFRGS, matheus.faria@ufrgs.br
}

\begin{abstract}
Resumo: O objetivo deste artigo é refletir sobre possibilidades de trabalho com um modelo matemático dinâmico em sala de aula e potencialidades do software GeoGebra nestes possíveis cenários. Para isso, consideramos um modelo matemático dinâmico para o problema de otimização da superfície do alvéolo de uma colmeia de abelhas. A discussão do potencial didático da proposta é realizada à luz de referencial teórico sobre modelagem matemática e sobre a utilização de tecnologias digitais, em especial a matemática dinâmica. Três possibilidades de trabalho com o modelo são abordadas: Aplicações, Análise de Modelos e Modelagem. Para cada uma delas, potencialidades do GeoGebra são elencadas, destacando-se aquelas relacionadas à visualização, dinamicidade e múltiplas representações do problema estudado.
\end{abstract}

Palavras-Chave: modelagem matemática, matemática dinâmica, alvéolo da colmeia, problemas de otimização

\section{Exploring a hive: a modelling proposal with GeoGebra 3D}

Abstract: The aim of this paper is to reflect on the possibilities of working with a dynamic mathematical model in the classroom and the potential of the GeoGebra software in these possible scenarios. For this, we consider a dynamic mathematical model for the problem of optimization of the honeycomb surface of a bee hive. The discussion of the didactic potential of the proposal is carried out in the light of a theoretical framework on mathematical modeling and on the use of digital technologies, especially dynamic mathematics. Three possibilities of working with the model are discussed: Applications, Model Analysis and Modeling. For each of them, GeoGebra's potentialities are listed, highlighting those related to visualization, dynamics and multiple representations of the studied problem.

Keywords: mathematical modelling, dynamic mathematics, alveolus of the hive, optimization problems

\section{Introdução}

As tecnologias digitais e, em especial, a matemática dinâmica, vêm impactando as formas de pensar e fazer matemática. Os recursos tecnológicos hoje proporcionam a construção de modelos matemáticos que até então ficavam restritos à representação em lápis e papel (Basso e Notare, 2015). Problemas complexos, cuja representação parecia inacessível, agora podem ser construídos e manipulados virtualmente.

Na disciplina Educação Matemática e Tecnologia, oferecida para licenciandos em Matemática da Universidade Federal do Rio Grande do Sul, cujo objetivo é desenvolver e aperfeiçoar competências e habilidades para a utilização de tecnologias digitais para 0 ensino e a aprendizagem da Matemática, os potenciais da matemática dinâmica são discutidos com os estudantes, a partir de construções em ambiente dinâmico acompanhadas de leituras, debates, seminários de apresentação e produções escritas. Nesse processo, os alunos refletem sobre as possibilidades e limitações do uso das tecnologias digitais na Educação Matemática. 
Neste artigo, apresentamos a produção de um dos estudantes da disciplina (terceiro autor desse artigo), que apresenta a elaboração de um modelo matemático dinâmico para otimizar a superfície do alvéolo de uma colmeia de abelhas, realçando as potencialidades do ambiente dinâmico para modelar e resolver problemas de otimização e indicando possibilidades de trabalho com esse modelo em sala de aula. A partir da discussão e análise do modelo apresentado pelo aluno, ampliamos esse debate, considerando referencial teórico da área.

Dessa forma, na seção 2 apresentamos os fundamentos teóricos que sustentam o artigo, discutindo aspectos da modelagem matemática e as potencialidades dos ambientes dinâmicos na compreensão de conceitos matemáticos. Na seção 3, discutimos a construção do modelo matemático dinâmico para o alvéolo no GeoGebra, apresentando o problema, sua interpretação e análise matemática e as etapas da construção. Finalmente, na seção 4, discutimos as possibilidades de utilização do modelo em sala de aula, apresentando as potencialidades do software GeoGebra em cada uma delas.

\section{Fundamentos Teóricos: Modelagem Matemática e Matemática Dinâmica}

Em Soares e Javaroni (2013) encontramos um debate acerca das possibilidades de trabalho em sala de aula com um modelo matemático. Com base na literatura da área e em exemplos de suas próprias pesquisas, as autoras apresentam três possíveis abordagens: a da Modelagem Matemática, a das Aplicações, e a da Análise de Modelos. No que segue, vamos retomar parte deste debate.

Iniciamos com a Modelagem ${ }^{1}$ que, na esfera da Educação Matemática, pode ser descrita, em linhas gerais, como sendo "uma abordagem em que os estudantes investigam um tema relacionado a outra área científica ou ao dia a dia e constroem um modelo matemático para lidar com um determinado problema vinculado ao tema investigado" (Soares e Javaroni, 2013, p.209). O processo de Modelagem, portanto, geralmente inicia com a escolha de um tema, um assunto que será estudado e investigado. No contexto deste tema, elege-se um problema, o qual será analisado considerando conceitos e ferramentas matemáticas que podem contribuir para o seu entendimento. Para Niss et al. (2007), ao fazer Modelagem é como se estivéssemos do lado de fora da Matemática e nos perguntássemos: "Onde posso encontrar alguma matemática para me ajudar com esse problema?" (Niss et al., 2007, p.10). Nesse sentido, para Niss et al. (2007) a Modelagem refere-se a um movimento na direção "realidade $\rightarrow$ matemática".

Esta descrição tenta abarcar processos que podem perpassar uma modelagem "genérica", considerando que existem diferentes perspectivas desta tendência em Educação Matemática. Quer dizer, dependendo da perspectiva de Modelagem assumida, haverá variação acerca de questões como: o entendimento acerca do que é e qual a importância de um modelo matemático; o sujeito responsável pela escolha do tema de investigação (professor(a) e/ou alunos); e até o "mundo" no qual o modelo em questão alcança (se é referente a um fenômeno real ou cibernético).

Outro movimento possível, segundo Niss et al. (2007, p.10), ocorre na direção "matemática $\rightarrow$ realidade". Este movimento corresponde à abordagem denominada pelos autores de Aplicações. Nesta abordagem, o processo inicia com a escolha de um conteúdo matemático e, a partir daí, busca-se um problema (ou mais de um) para o qual esse conteúdo se aplica, isto é, com o qual é possível construir um modelo que ajude a compreender o problema. Neste caso, um modelo pode ser usado para ilustrar uma aplicação do conteúdo; ou então os alunos podem se engajar na criação de um modelo, já conhecendo o conceito matemático a ser utilizado neste processo.

\footnotetext{
${ }^{1}$ Para evitar repetições, iremos utilizar o termo Modelagem para nos referir à Modelagem Matemática.

2 "Where can I find some mathematics to help me with this problem?"
} 
Finalmente, uma terceira abordagem possível é a Análise de Modelos (Soares, 2012). Conforme debatido em Soares (2012) e em Soares e Javaroni (2013), esta abordagem tem semelhanças e diferenças com a Modelagem e também com as Aplicações. Aqui os alunos iniciam o trabalho a partir de um modelo matemático já existente para algum problema de outra área científica ou do dia a dia. O trabalho tem como um de seus objetivos introduzir conceitos matemáticos novos para os alunos, conceitos estes que estão relacionados ao modelo matemático escolhido e que são debatidos com os alunos a partir do estudo e da análise do modelo.

A Análise de Modelos propõe uma ênfase no entendimento qualitativo do modelo, de forma relacionada ao fenômeno modelado. Uma das motivações por trás dessa abordagem é que os alunos tenham a oportunidade de trabalhar com modelos mais acurados para a situação estudada. Modelos com essas características algumas vezes apresentam um conteúdo matemático ainda avançado para os alunos ou para o qual uma solução analítica não existe. Por causa disso, o uso de tecnologias digitais pode ter um papel central nessa abordagem. Por exemplo, em Soares e Borba (2014), os autores argumentam sobre o papel central do software para que alunos de Biologia cursando Cálculo Diferencial e Integral (CDI) conduzissem a análise de um modelo matemático para a transmissão da malária e, com base na mesma, construíssem alguns conceitos previstos na ementa da disciplina.

Dentre os vários conceitos matemáticos de uma disciplina de CDI, o conceito de função pode ser considerado como uma ferramenta matemática que ajuda a entender os fenômenos do universo a partir da análise das relações de interdependência entre grandezas, que são intrínsecas a esses fenômenos. O CDI é o ramo da Matemática que busca estudar em profundidade e com precisão como as grandezas se relacionam e como ocorrem as relações entre as variáveis de um fenômeno. Portanto, entender que a variação de uma grandeza depende da variação de outra é um aspecto crucial para a compreensão do conceito de função e, em especial, para o estudo do CDI, mas que, segundo Rezende et al (2012, p.75-76), "se torna incompleto do ponto de vista epistemológico, se não estudamos como ocorre esta variação, isto é, se não conseguimos dar qualidade e quantificar este processo de variação".

Nesse sentido, proporcionar um estudo dinâmico do comportamento funções pode contribuir para um entendimento amplo desse conceito, com ênfase na ideia de variabilidade, e os softwares de matemática dinâmica podem contribuir para esse processo, em particular o software GeoGebra. Com as possibilidades de construção, exploração, manipulação e análise da situação proposta a partir do modelo dinâmico e suas diferentes representações no ambiente de matemática dinâmica, entendemos que estudante e tecnologia digital estabelecem um movimento contínuo de agir e pensar, no qual o GeoGebra e suas ferramentas tornam-se uma extensão do pensamento do indivíduo. Dessa forma, o ambiente dinâmico do GeoGebra interfere e modifica as formas de pensar do indivíduo, impulsionando um avanço na compreensão proporcionado pelas representações agora acessíveis nesses ambientes (Basso e Notare, 2015).

\section{Matemática dinâmica na construção de modelos matemáticos}

A disciplina de Educação Matemática e Tecnologia tem como objetivo desenvolver competências e habilidades no futuro professor de Matemática para a utilização de tecnologias digitais no ensino e na aprendizagem da Matemática. Uma das tarefas da disciplina foi propor aos licenciandos a análise e discussão das possibilidades de utilização do ambiente de matemática dinâmica GeoGebra para trabalhar com problemas de otimização.

Trazemos para debate e análise a produção do terceiro autor deste artigo, na época aluno da disciplina, para refletir sobre as possibilidades de entrelaçamento da modelagem 
matemática com a matemática dinâmica na sala de aula. A partir da produção e das reflexões desse futuro professor de Matemática, discutimos as potencialidades do ambiente dinâmico GeoGebra para a resolução de um problema de otimização em conjunto com as possibilidades de trabalho com esse modelo em sala de aula.

\subsection{O problema da colmeia}

O aluno Matheus, desafiado pela tarefa proposta em aula, apresentou a construção do modelo matemático dinâmico inspirado no problema de otimização proposto por Stewart (2013, p.301), que traz o seguinte enunciado:

Em uma colmeia, cada alvéolo é um prisma hexagonal regular, aberto em uma extremidade com um ângulo triédrico na outra extremidade. Acredita-se que as abelhas formam esses alvéolos de modo a minimizar a área da superfície, usando assim uma quantidade mínima de cera na construção. O exame desses alvéolos mostrou que a medida do ângulo do ápice $\theta$ é surpreendentemente consistente. Baseado na geometria do alvéolo, pode ser mostrado que a área da superfície $S$ é dada por

$$
S=6 \operatorname{sh}-\frac{3}{2} s^{2} \operatorname{cotg}(\theta)+\frac{3 \sqrt{3}}{2} s^{2} \operatorname{cossec}(\theta),
$$

onde $s$, o comprimento dos lados do hexágono, e h, a altura, são constantes.

Então, são solicitados os seguintes itens, junto a uma figura do alvéolo:

(a) Calcule $\frac{d S}{d \theta}$.

(b) Que ângulo as abelhas deveriam preferir?

(c) Determine a área mínima da superfície do alvéolo (em termos de s e h).

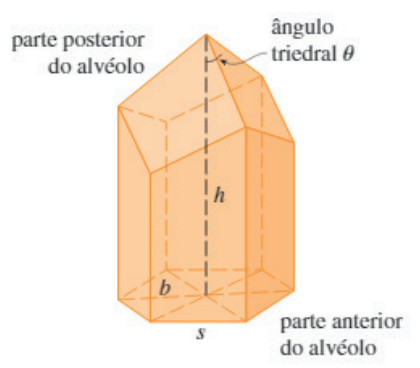

Figura 1. Enunciado do problema e representação geométrica do alvéolo.

Fonte: Stewart (2013)

Analisando a figura ilustrada em Stewart (2013) e a expressão algébrica que determina a superfície, também apresentada no enunciado, Matheus identificou que a constante $h$ destacada no desenho como altura do alvéolo em si, na realidade é a medida das alturas dos trapézios laterais, o que gera inconsistência (no enunciado não está explícito qual dessas duas alturas é $h$ ).

Ao observar a Figura 1, é possível ter uma ideia da forma do alvéolo para certo ângulo $\theta$. Uma vez que no papel, o ângulo $\theta$ está fixado junto com as constantes $s$ e $h$ (e o $h$ da figura, em particular, não é o mesmo da função de superfície fornecida), uma possibilidade para resolver o problema consiste em deixar de lado os itens do problema por ora e abordar a situação descrita de uma forma computacional, utilizando a matemática dinâmica para construir uma situação na qual $s$ e $h$ estejam fixados adequadamente e $\theta$ varie. Logo, trata-se de construir um modelo matemático dinâmico para o problema em questão.

No caso proposto por Matheus, o modelo buscado é geométrico. Ele consiste em uma adaptação de uma situação real (a organização da colmeia). Stewart (2013) apresenta o problema inicial, mas é possível complementar as informações, como medidas-padrão das dimensões, de modo a potencializar a precisão do modelo. Especificamente, o enunciado do problema não apresenta informações acerca do fundo do alvéolo além do ângulo triédrico. Mas, de acordo com Calebante (2012), o fundo de cada alvéolo é constituído de três losangos iguais e também serve como fundo para outros alvéolos, pois a colmeia é arranjada em favos com duas fileiras de alvéolos.

A partir dessas informações, o Matheus chega a uma ideia do que deve ser contemplado no modelo: um prisma hexagonal aberto em uma extremidade e seccionado 
por três planos em outra extremidade, os quais contém os losangos. A inclinação desses planos deve depender do ângulo triédrico. A seguir, apresentamos a etapa de construção do modelo no GeoGebra, realizada por Matheus.

\subsection{Construção do modelo do alvéolo no GeoGebra}

Como já mencionado, para construir o modelo, Matheus escolheu o GeoGebra. A possibilidade de construir pontos e gráficos que são dependentes do modelo geométrico construído, de modo que a movimentação de elementos variáveis no modelo provoque a alteração síncrona desses pontos na janela gráfica, proporcionando uma exploração qualitativa da relação de dependência entre as variáveis, justifica a escolha desse software.

Dentre os vários recursos disponíveis, o GeoGebra possui a ferramenta controle deslizante, que permite criar variáveis no software. Matheus iniciou sua construção com essa ferramenta, criando três controles para as três variáveis do problema ( $s, h$ e $\theta)$. Dessa forma, é possível explorar qualitativamente a situação apresentada, analisando as relações entre as variáveis, de modo que, ao alterar seus valores, é possível observar simultaneamente a variação do modelo geométrico, da relação algébrica e do gráfico, possibilitando estudar características locais e variacionais do comportamento da função.

A partir dos controles deslizantes criados, Matheus utilizou ferramentas do GeoGebra para construir um prisma hexagonal regular cuja a altura é determinada pela variável $h$, definida por um dos controles deslizantes. O próximo passo de Matheus foi encontrar uma estratégia para construir o ângulo triédrico $\theta$. Nesse momento, conceitos geométricos entram em jogo, o que torna a modelagem do problema mais interessante. Matheus usou a ferramenta segmento para traçar uma diagonal da base que não passe pelo centro do hexágono. Também determinou o ponto médio dessa diagonal, e finalizou construindo um plano perpendicular à diagonal, contendo o ponto médio (Figura 2).

Mas qual a importância desse plano perpendicular na construção de Matheus? Note que até agora, o controle deslizante do ângulo não foi utilizado. Assim, Matheus recorreu a um recurso exclusivo da janela 3D: clicou no plano desejado (o plano que construiu e está ilustrado na Figura 2) com o botão direito do mouse e criou uma vista 2D do mesmo, conforme podemos ver na Figura 3.

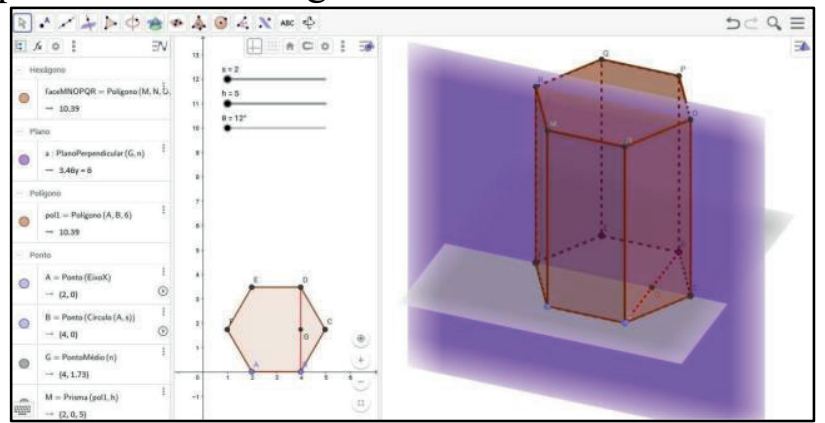

Figura 2. Construção parcial, sendo a janela 2D equivalente ao plano $x \mathrm{O} y$ da janela 3D.

Nesse plano, Matheus usou o segmento RO e a reta paralela a $\mathrm{CO}$, encontrando o ponto $\mathrm{H}$ em RO para obter o vértice do ângulo. Então, utilizou a ferramenta ângulo com amplitude fixa, o ponto médio da diagonal e o vértice do ângulo, criando o ângulo com amplitude $\theta$. O software executa a rotação $\theta$ do ponto médio em torno do vértice, e gera um novo ponto a partir dessa rotação. Matheus traçou uma reta que passa pelo vértice do ângulo e por esse ponto para determinar o ponto de intersecção (que chamou de pontochave) dessa reta com a aresta do prisma. Finalmente, refletiu o ponto-chave em relação ao vértice do ângulo. O segmento que vai do ponto-chave até seu reflexo é uma diagonal de um dos três losangos que formam o fundo do alvéolo. 


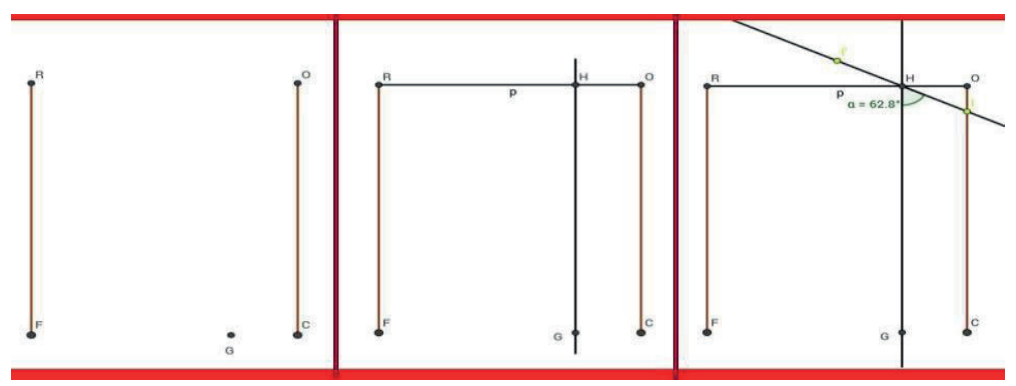

(a)

(b)

(c)

Figura 3. (a) Vista 2D do plano. (b) Obtenção do vértice do ângulo $\theta$. (c) Construção do ponto-chave.

O ponto-chave é crucial, pois a partir dele é possível determinar a altura do alvéolo e os planos nos quais se encontram os losangos do fundo do alvéolo. Usando a ferramenta plano por três pontos, Matheus determinou os vértices do alvéolo, conforme ilustra a Figura 4.

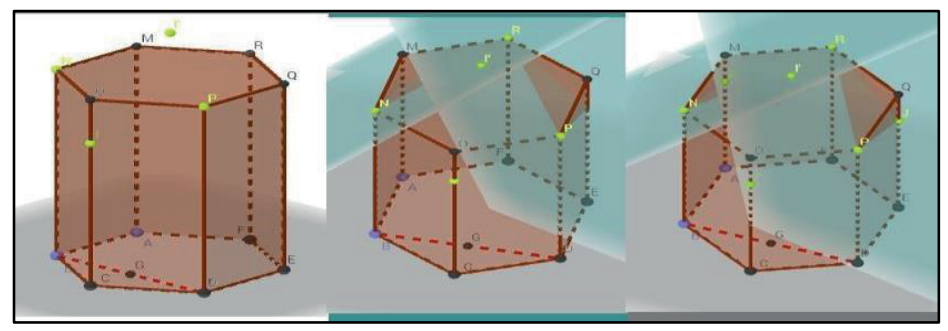

(a)

(b)

(c)

Figura 4. (a) Vértices de um losango. (b) Construção dos planos que contém os outros dois losangos. (c) Determinação dos últimos vértices do alvéolo.

De posse de todos os vértices do alvéolo, Matheus passou à construção dos polígonos que compõem a superfície do alvéolo. Na Figura 5, temos o modelo do alvéolo final construído pelo aluno. Fixados $s=3$ e $h=8$, temos na figura alvéolos com ângulos triédricos de 45, 60 e 90 graus, respectivamente.

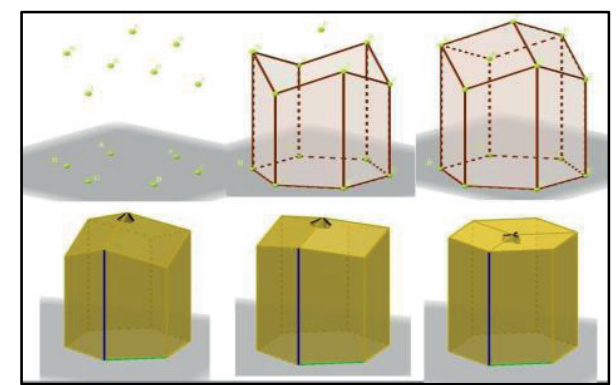

Figura 5. Construção da superfície e variação de acordo com o ângulo triédrico.

É válido destacar que essa forma de construção do alvéolo não é única. Fatores como as ferramentas utilizadas e a ordem de construção dos objetos são capazes de contribuir para a determinação de diferentes caminhos - ou seja, para a exploração de diferentes conceitos - que levem à obtenção do mesmo modelo.

\section{Possibilidades de uso do modelo em sala de aula}

Nesta seção, discutimos as possibilidades de uso da modelagem dinâmica em sala de aula, para o estudo de conceitos de Cálculo Diferencial, utilizando como exemplo o modelo do alvéolo construído por Matheus. Para tanto, em cada subseção, consideramos como este modelo poderia ser trabalhado em sala de aula segundo as possibilidades apresentadas na seção 2. Além disso, procuramos identificar as potencialidades do GeoGebra em cada um destes possíveis cenários. 


\subsection{Perspectiva das Aplicações}

Conforme apresentamos na seção 2, uma das abordagens possíveis para o uso do problema de otimização do alvéolo em sala de aula é a das Aplicações. Segundo Soares e Javaroni (2013, p.14) a ideia central dessa abordagem consiste em "iniciar com a definição de um conteúdo matemático para depois seguir com a apreciação de uma situação ou problema para o qual aquele conteúdo seria utilizado".

Em Stewart (2013), o problema é proposto no capítulo intitulado "Aplicações da Derivação". Em particular, conteúdos de Cálculo como funções trigonométricas, derivada, pontos críticos, entre outros, são apresentados antes do problema. Deste modo, podemos utilizar o modelo construído em uma disciplina de CDI sob a perspectiva das Aplicações, apresentando o modelo dinâmico já construído para os alunos e usando-o para explorar uma situação real na qual os conteúdos matemáticos trabalhados anteriormente são aplicados.

Um dos potenciais de utilizar matemática dinâmica é a possibilidade de visualizar e explorar o gráfico da função superfície e, a partir dele, obter o gráfico de sua derivada, para analisar qualitativamente o comportamento da variação da função e da relação entre as variáveis. Na Figura 6, temos representados esses dois gráficos (função e sua respectiva derivada) em uma janela de visualização secundária.

A partir de explorações dinâmicas no modelo construído, ao que tudo indica, o ângulo que otimiza a construção das abelhas é de aproximadamente 55 graus. É possível observar a inclinação quase nula da reta tangente ao gráfico em $\mathrm{S}$. Com o modelo dinâmico, os estudantes podem manipular o controle deslizante $\theta$ e explorar múltiplas possibilidades para o ângulo do alvéolo e, de forma simultânea, analisar o comportamento gráfico da função que descreve sua superfície, podendo estabelecer relações entre a medida de $\theta$ e o valor da superfície do alvéolo.

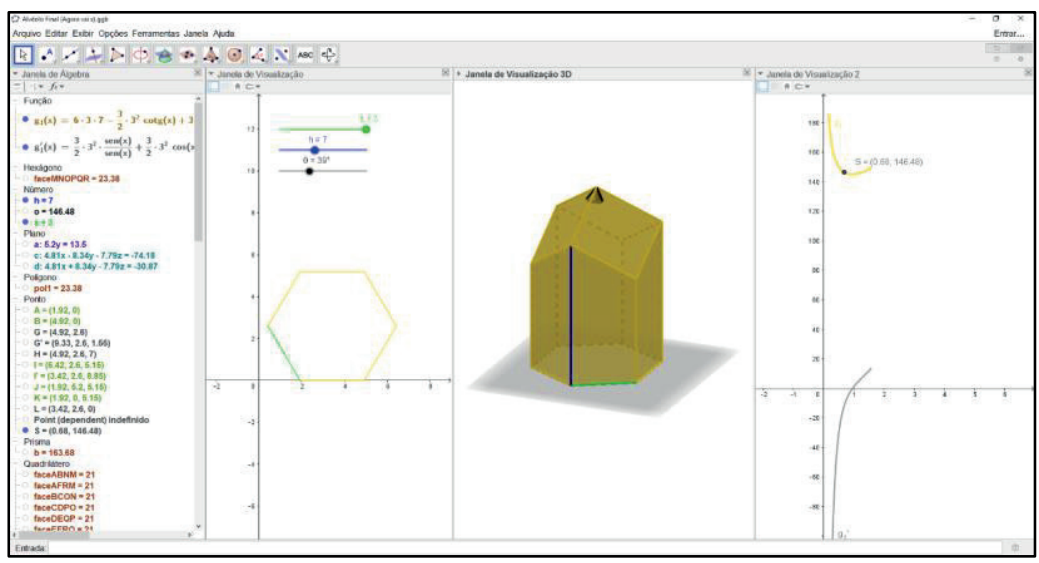

Figura 6. Gráficos da função superfície e de sua derivada.

Porém, a exploração do modelo não precisa (nem deve) parar por aqui. Ter uma ideia visual e dinâmica da solução do problema pode encorajar o estudante a uma compreensão mais ampla da situação explorada (e dos conceitos de função, taxa de variação e ponto crítico). Compreender as relações entre a variação e a escolha do ângulo e o comportamento gráfico da função da superfície do alvéolo devem ser estimuladas. Perguntas que conduzam os estudantes a explorar o controle deslizante, analisar o comportamento da função e compreender a relação de dependência entre as variáveis proporcionam um entendimento da situação, que vai além da pura resolução algébrica ou numérica. Finalmente, após as conjecturas sobre a possível solução do problema, podese passar para a etapa de resolução algébrica, na qual será possível confrontar os resultados, caminhando para o entendimento global do modelo e consolidação dos conceitos matemáticos envolvidos. 
Percebe-se, nesse sentido, que as respostas de questões fechadas como os itens (a), (b) e (c) do problema inicial podem ficar mais fáceis de visualizar (talvez até triviais, partindo do pressuposto de que os alunos já viram o conteúdo matemático envolvido no problema). O modelo ainda permite a exibição de resultados numéricos, o que seria uma particularização do item (c), que solicita a superfície mínima em termos de $s$ e $h$. Porém, quando estamos utilizando recursos tecnológicos, devemos ir além do que os problemas propõem, buscando uma compreensão qualitativa da situação, e não apenas encontrar respostas para as questões propostas.

\subsection{Perspectiva da Análise de Modelos}

Agora, vamos supor que esse modelo seja apresentado aos alunos no primeiro dia de aula (ou em algum momento específico da disciplina). Dessa forma, o modelo pode ser utilizado para introduzir conceitos novos aos alunos. Conforme abordamos na seção 2, Soares e Javaroni (2013) chamam esse tipo de trabalho de Análise de Modelos.

O modelo discutido nesse artigo, particularmente, é bastante abrangente, ao passo que vários conhecimentos típicos do CDI podem ser construídos a partir dele, a saber: funções trigonométricas, gráficos de funções, domínio e imagem de uma função, derivada de uma função trigonométrica, máximos e mínimos, entre outros.

É interessante notar a relação entre essa perspectiva e o uso de tecnologias digitais. Na perspectiva das Aplicações, o modelo do alvéolo feito no GeoGebra pode ter um caráter mais complementar, servindo principalmente à etapa de validação dos dados. $\mathrm{Na}$ Análise de Modelos, o alvéolo pode ter seu caráter essencial, pois o trabalho com o software é o que possibilita aos alunos a construção do conhecimento matemático. Conforme Soares e Javaroni (2013, p.16) afirmam, "um exemplo das possibilidades oferecidas pelas tecnologias à Análise de Modelos é a oportunidade de trabalhar com modelos envolvendo conceitos matemáticos ainda desconhecidos para os alunos.".

Um trabalho dentro desta perspectiva, iniciaria com a proposição do problema aos alunos, porém sem a indicação dos itens (a), (b) e (c). Em um primeiro momento os alunos seriam convidados a identificar que hipóteses e simplificações estão por trás da elaboração do modelo, assim como a entender seu processo de construção. Um próximo passo seria explorar os parâmetros do problema, por meio de seus controles deslizantes: Que mudanças a variação desses parâmetros geram no modelo? O que elas significam? Que informações nos dizem sobre o fenômeno? Neste momento, a dinamicidade e a interligação entre as diferentes representações fornecidas pelo software têm papel fundamental. Conforme Soares (2012), esse tipo de análise confere ao software um papel de laboratório digital, pois permite a modificação das condições de ocorrência do fenômeno e, portanto, se assemelha a uma exploração feita em um laboratório.

Finalmente, na sequência seriam apresentadas tarefas de caráter investigativo mais específicas, voltadas para a construção de conceitos matemáticos relacionados ao modelo. Por exemplo, poderíamos propor uma tarefa investigativa que tivesse como objetivo a construção do conceito de derivada a partir de um estudo do comportamento da função superfície. Para isso, de forma semelhante ao que foi proposto em Soares (2012), poderse-ia sugerir a investigação das taxas de variação média e instantânea da função, assim como a interpretação acerca da informação que essas taxas fornecem sobre o fenômeno. Neste processo, o software estaria fornecendo acesso aos dados para poder conduzir este estudo.

De forma semelhante ao proposto na seção anterior, um estudo qualitativo dos gráficos da função superfície e de sua derivada também poderia ser desenvolvido. Ainda, o problema de minimizar a superfície do alvéolo poderia ser explorado na sequência, a partir da análise qualitativa do modelo e da variação dos parâmetros com o GeoGebra, antes mesmo de os alunos aprenderem a determinar algebricamente a derivada da função. 


\subsection{Construção do modelo pelos alunos}

Ao observar as duas perspectivas anteriores de uso do modelo, percebemos que ele é apresentado já construído para os alunos, proporcionando a exploração a partir de uma construção já existente. Mas uma terceira possibilidade trata de investigar rigorosamente as propriedades geométricas que o objeto a ser modelado possui. Quais são as figuras geométricas que compõem o alvéolo? Que propriedades geométricas e regularidades possuem? Quais as relações entre as figuras geométricas e as variáveis $s, h$ e $\theta$ ? Quais as invariantes das figuras geométricas quando manipulamos essas variáveis pelos controles deslizantes? Essas são perguntas cruciais para realizarmos a construção de um modelo coerente e que podem ser provocativas para desencadear a construção pelos alunos.

Mais precisamente, podemos propor aos alunos a construção do alvéolo, de modo a deduzir, a partir de conceitos geométricos, a equação de sua superfície em termos do ângulo $\theta$, em vez de simplesmente tomar a função superfície dada por Stewart, por exemplo.

Gravina (1996) comenta sobre um aspecto que dificulta o aprendizado de geometria para os alunos, que é o fato de eles confundirem propriedades do desenho com propriedades do objeto. Em geral, isso ocorre em função de objetos prototípicos que são feitos no papel, como retângulos com os lados paralelos à folha, alturas em triângulos acutângulos, e assim por diante. Esses protótipos, por repetição excessiva, acabam sendo tomados como as únicas representações possíveis, o que gera inconsistências no aprendizado de geometria a longo prazo.

E isso ocorre no problema discutido neste artigo. Na Figura 1, que ilustra o enunciado do livro, temos a representação de um alvéolo prototípico (ou quase isso, já que a forma do alvéolo, diferente de um prisma hexagonal regular reto ou oblíquo, não é comum de ser encontrada em livros). Ocorre que a constante $h$, no desenho apresentado pelo livro, é a altura do alvéolo em si. Porém, se construirmos o modelo dinâmico no GeoGebra 3D com essa propriedade, pode ser verificado que o valor da superfície na função dada pelo enunciado é diferente do valor da superfície da figura, $\operatorname{com} \theta$ variando igualmente para os dois. Ou seja, o objeto que obedece a fórmula dada por Stewart não é o mesmo que está representado no desenho.

Esse é um exemplo de inconsistência que dificilmente seria revelada e corrigida com uma verificação do modelo construído, mas sim com uma tentativa de reconstrução rigorosa do mesmo, na qual a constante $h$ "verdadeira" é a medida da base maior dos trapézios laterais do alvéolo. Dessa maneira, uma possível forma de trabalhar essa questão em um curso de Geometria seria propondo aos alunos a construção do modelo geométrico no GeoGebra, a partir de suas propriedades geométricas.

\section{Considerações Finais}

$\mathrm{Na}$ vida real, as abelhas não têm noções de cálculo diferencial, nem possuem conhecimentos teóricos sobre geometria euclidiana. Apesar disso, elas conseguem ser surpreendentemente econômicas na quantidade de cera que compõe suas colmeias. Destacamos que já foram feitas medidas do ângulo $\theta$ na prática, e os ângulos triédricos de colmeias reais não costumam apresentar erro maior do que $2^{\circ}$ em relação ao ângulo ótimo obtido pelos conceitos do Cálculo. Diante dessa aproximação, podemos concluir que a modelagem com matemática dinâmica apresenta potencial matemático e didático para abordar o problema, tanto para verificar a aproximação quanto para proporcionar compreensão da situação como um todo.

É válido observar que as três perspectivas de trabalho apresentadas aqui não são as únicas possíveis, pois elas estão inseridas em um contexto acadêmico, pensadas para o Ensino Superior. Mas não é difícil pensar que a construção do alvéolo com os três parâmetros fixados - por exemplo - pode-se constituir como uma atividade rica e 
interessante no contexto de aprendizagem de Geometria Espacial ou da análise de comportamento de funções e relações qualitativas entre variáveis na Educação Básica. Dessa forma, fica a reflexão para pensar em outras finalidades para o modelo apresentado e discutido nesse artigo, para além das questões que o originaram.

A partir do entendimento de um futuro professor de Matemática sobre as possibilidades de utilização das tecnologias digitais, em especial da matemática dinâmica, para trabalhar com estudantes do ensino médio ou superior, discutimos diferentes abordagens da modelagem matemática. Para cada uma delas, buscamos identificar potencialidades do GeoGebra, as quais se mostraram relacionadas à visualização, dinamicidade e múltiplas representações do problema estudado.

As discussões e análise do modelo realizadas pelo aluno, refletindo sobre as possíveis formas de abordagem em sala de aula, apontam para a importância de desenvolver, nos futuros professores de Matemática, competências e habilidades para a utilização de tecnologias digitais para o ensino e a aprendizagem da Matemática.

\section{Referências}

BASSO, M., NOTARE, M. Pensar-com Tecnologias Digitais de Matemática Dinâmica. In:

Revista Novas Tecnologias na Educação - RENOTE, Porto Alegre: UFRGS, v.13, n.2, 2015.

CALEBANTE. Abelhas Sabem Cálculo Diferencial. Disponível em $<$ http://calebante.blogspot.com.br/2012/02/abelhas-sabem-calculo-diferencial.html> Acesso em: 18 dez. 2017.

GEOGEBRA ONLINE. Disponível em <https://www.geogebra.org/classic> Acesso em: 20 dez. 2017.

GRAVINA, M. Geometria Dinâmica uma nova abordagem para o aprendizado da geometria. In: Anais do VII Simpósio Brasileiro de Informática na Educação, p.1-13, Belo Horizonte, nov. 1996.

GRAVINA, M.; CONTIERO, L. Modelagem com o GeoGebra: uma possibilidade para a educação interdisciplinar? In: RENOTE: Revista Novas Tecnologias na Educação, Porto Alegre, v.9, n. 1, jul. 2011.

HALL, J., LINGEFJARD, T. Mathematical Modeling: Applications with GeoGebra. Hoboken, New Jersey: John Wiley \& Sons, 2017.

NISS, M. Prescriptive modelling - challenges and opportunities. In: STILLMAN, G. A; BLUM, W.; BIEMBENGUT, M. S. (Eds.) Mathematical Modelling in Education Research and Practice: cultural, social and cognitive influences. Cham: Springer, 2015. p.67-80.

REZENDE, W.; PESCO, D.; BORTOLOSSI, H. Explorando aspectos dinâmicos no ensino de funções reais com recursos do GeoGebra. In: $1^{\mathrm{a}}$. Conferência Latino Americana de GeoGebra, 2012, p. 74-89.

SOARES, D. S. Uma Abordagem Pedagógica Baseada na Análise de Modelos para Alunos de Biologia: qual o papel do software? 2012. 341f. Tese (Doutorado em Educação Matemática) - Instituto de Geociências e Ciências Extas, Universidade Estadual Paulista, Rio Claro, SP, 2012.

SOARES, D. S. Model Analysis with Digital Technologies: a "hybrid approach". In: STILlMAN, G. A.; BLUM, W.; BIEMBENGUT, M. S. (Eds.) Mathematical Modelling in Education Research and Practice: cultural, social and cognitive influences. Cham: Springer, 2015. p. 453-463.

SOARES, D. S.; BORBA, M. C. The role of software Modellus in a teaching approach based on Model Analysis. ZDM, Heidelberg, Alemanha, v.46, n.4, p. 575-587, ago. 2014.

SOARES, D.S.; JAVARONI, S.L. Análise de Modelos: possibilidades de trabalho com modelos matemáticos em sala de aula. In: BORBA, M.C.; CHIARI, A. (Org) Tecnologias Digitais e Educação Matemática, São Paulo: Editora Livraria da Física, 2013. p.195-219.

STEWART, James. Cálculo, V.1. $7^{a}$ edição. São Paulo: Cengage Learning, 2013. 